\title{
Unsupervised Factory Activity Recognition with Wearable Sensors Using Process Instruction Information
}

\author{
XIA QINGXIN, Osaka University, Japan \\ ATSUSHI WADA, Toshiba Corporation, Japan \\ JOSEPH KORPELA, Osaka University, Japan \\ TAKUYA MAEKAWA*, Osaka University, Japan \\ YASUO NAMIOKA, Toshiba Corporation, Japan
}

\begin{abstract}
This paper presents an unsupervised method for recognizing assembly work done by factory workers by using wearable sensor data. Such assembly work is a common part of line production systems and typically involves the factory workers performing a repetitive work process made up of a sequence of manual operations, such as setting a board on a workbench and screwing parts onto the board. This study aims to recognize the starting and ending times for individual operations in such work processes through analysis of sensor data collected from the workers along with analysis of the process instructions that detail and describe the flow of operations for each work process. We propose a particle-filter-based factory activity recognition method that leverages (i) trend changes in the sensor data detected by a nonparametric Bayesian hidden Markov model, (ii) semantic similarities between operations discovered in the process instructions, (iii) sensor-data similarities between consecutive repetitions of individual operations, and (iv) frequent sensor-data patterns (motifs) discovered in the overall assembly work processes. We evaluated the proposed method using sensor data from six workers collected in actual factories, achieving a recognition accuracy of $80 \%$ (macro-averaged F-measure).
\end{abstract}

CCS Concepts: • Human-centered computing $\rightarrow$ Ubiquitous and mobile computing theory, concepts and paradigms; - Mathematics of computing $\rightarrow$ Sequential Monte Carlo methods; Time series analysis;

Additional Key Words and Phrases: Activity recognition, wearable sensor, factory work

ACM Reference Format:

Xia Qingxin, Atsushi Wada, Joseph Korpela, Takuya Maekawa, and Yasuo Namioka. 2019. Unsupervised Factory Activity Recognition with Wearable Sensors Using Process Instruction Information. Proc. ACM Interact. Mob. Wearable Ubiquitous Technol. 3, 2, Article 60 (June 2019), 23 pages. https://doi.org/10.1145/3328931

\section{INTRODUCTION}

Due to the recent proliferation of smart sensing devices such as smartphones, smart watches, and smart glasses, human activity recognition using sensor data obtained from body-worn smart devices has been actively studied in the ubicomp and wearable-computing research communities [15, 28, 35, 39]. Activity recognition using these

${ }^{*}$ This is the corresponding author

Authors' addresses: Xia Qingxin, Osaka University, Graduate School of Information Science and Technology, Suita, Osaka, 5650871, Japan; Atsushi Wada, Toshiba Corporation, Corporate Manufacturing Engineering Center, Yokohama, Kanagawa, 2350017, Japan; Joseph Korpela, Osaka University, Graduate School of Information Science and Technology, Suita, Osaka, 5650871, Japan; Takuya Maekawa, takuya.maekawa@acm.org,maekawa@ist.osaka-u.ac.jp, Osaka University, Graduate School of Information Science and Technology, Suita, Osaka, 5650871, Japan; Yasuo Namioka, Toshiba Corporation, Corporate Manufacturing Engineering Center, Yokohama, Kanagawa, 2350017, Japan.

ACM acknowledges that this contribution was authored or co-authored by an employee, contractor, or affiliate of the United States government. As such, the United States government retains a nonexclusive, royalty-free right to publish or reproduce this article, or to allow others to do so, for government purposes only.

(c) 2019 Association for Computing Machinery.

$2474-9567 / 2019 / 6-A R T 60 \$ 15.00$

https://doi.org/10.1145/3328931

Proc. ACM Interact. Mob. Wearable Ubiquitous Technol., Vol. 3, No. 2, Article 60. Publication date: June 2019. 


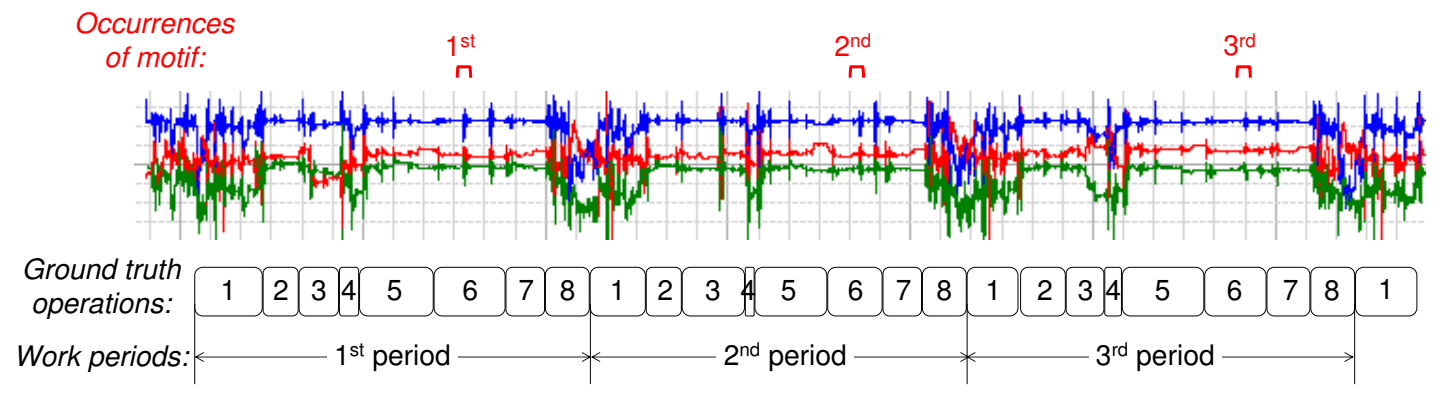

Fig. 1. Example acceleration data collected from a worker. Red, green, and blue lines show $x^{-}, y^{-}$, and z-axis data, respectively. The rectangles below show the ground truth labels of the operations. The duration of each operation period is about 120 s. Each work period consists of eight operations, which are defined in the process instructions. Red brackets show the occurrences of a motif detected by the proposed method.

wearable sensors can be applied to a variety of applications in both industrial settings, e.g., quality assurance for assembly workers, and home settings, e.g., analysis of daily living conditions of elderly patients. This paper focuses on the application of activity recognition in an industrial setting, namely in the analysis of assembly work by factory workers using data captured by wrist-worn acceleration sensors.

Many factories employ a line-production system in which each product passes through the same sequence of work processes in series. Often included amongst these processes is assembly work conducted manually by factory workers, where each worker repetitively performs a set of predefined processes with each process consisting of a sequence of operations, such as setting a board on a workbench and screwing parts onto the board. Such assembly work by factory workers still constitutes the core of line-production systems, making the improvement of assembly work one of the most important aspects of increasing productivity in these systems $[2,20]$.

The goal of this work is to recognize the individual operations conducted during an overall production process by using wearable sensor data. More specifically, we identify the starting and ending times of each individual operation through analysis of the sensor data. Having detected the timing of the operations, we can then support many useful applications, such as monitoring the status of workers and detecting outlying operations. Such automated monitoring systems for assembly-work activities are in high demand by manufacturers, since lineproduction systems often employ large numbers of workers and it can be difficult for line managers to monitor all their workers simultaneously.

Previous work in the ubicomp community has applied supervised learning techniques to recognize these types of actions (operations) using wearable sensor data. However, collecting labeled training data such as is required by these supervised approaches can be prohibitively costly when applied to assembly-work activities due to the following issues:

(1) Each worker may perform a different operation process, necessitating training data collection for every worker.

(2) The operations themselves may be modified frequently (e.g., weekly or monthly) due to frequent revisions of the production system, necessitating training data collection on a regular basis.

This study addresses this shortfall in previous supervised techniques by attempting to recognize line-production operations in an unsupervised manner.

Figure 1 shows example sensor data collected from a worker. As shown in the figure, a typical work process is iterated several times, with each iteration of the process (i.e., work period) comprised of a sequence of operations. 
In this example, we have eight operations per work period, iterated three times. The goal of this study is to identify the starting and ending times for each operation, as well as to estimate class labels for the operations. However, as can be seen in the figure, it is difficult to identify the sensor data segments corresponding to different operations solely using sensor data. The main feature of the proposed method is to leverage information included in process instructions in conjunction with the sensor data. Process instructions are documents that describe the flow of the operations included in each work process, as well as give detailed instructions for each operation. In this study, we assume that these instructions include information about the standard duration of each operation in a process, which we can use when identifying the starting and ending times of the individual operations. However, in practice the actual duration of each operation will differ from one work period to the next as shown in Figure 1, meaning that the process instructions alone cannot be used to accurately identify the boundaries between operations.

In this work, we employ a particle filter to robustly identify the boundaries of the operations despite the variance in their durations. A particle filter [10] is usually used to track an object in the computer vision research community. We employ a particle filter to robustly track the starting times of operations that appear non-linearly. We propose the following techniques to enable the precise unsupervised recognition of assemblywork operations based on a particle filter:

(1) We note that the transition points between operations in a work period may contain noticeable trend changes in the sensor data, e.g., in Figure 1 we can see a dramatic change in the characteristics of the sensor data at each ending of the first operation. We can identify candidates for these transition points based on the trend changes detected by a nonparametric Bayesian hidden Markov model (HMM) and then determine the best sequence of transition points from these candidates by matching them to the standard operation durations using a particle filter.

(2) We observe that operations with semantically similar descriptions in the working instructions may have similar sensor data. For example, description text of "screwing component A" operation can be semantically similar with that of "screwing component B" operation. And since both operations contain a screwing action, we expect that their sensor data will also be similar. Therefore, we can compare the similarities in the operations' sensor data for each candidate segmentation with their semantic similarities in order to identify the best candidate segments of the operations.

(3) It is likely that the sensor data of an operation from one work period will be similar with data from the same operation in recent periods from the same worker. Thus, we can calculate the sensor data similarity for an operation across different work periods in order to identify the correct sensor data segments for the operation.

The research contributions of this study are summarized as follows.

- We propose an unsupervised factory-activity recognition method for wearable sensors.

- The proposed method leverages information extracted from process instructions as well as sensor data analysis to precisely recognize activities in an unsupervised manner.

- To our knowledge, this is the first study on recognizing factory activities without the use of labeled training data.

- We evaluate the proposed method using sensor data collected from six workers in actual factories. Using this data, the proposed method achieved 80\% recognition accuracy (F-measure).

In the rest of this paper, we first review activity recognition studies that use wearable sensors including factory-activity recognition studies. We then present the proposed activity recognition method and evaluate the method using sensor data collected in actual factories. 


\section{RELATED WORK}

Due to the recent advance in sensing technologies, a variety of sensors are used to recognize human activities such as inertial sensors [6, 36], microphones [9, 14], cameras [26, 29, 33], and magnetic sensors [24, 25]. In particular, human activity recognition using acceleration sensors is a core topic in the ubicomp community, with many prior examples of it being conducted using supervised machine learning techniques that rely on labeled training data $[8,18,22,23]$. Along with these supervised-learning techniques, several studies have also been done on reducing the effort required to collect the labeled training data needed for supervised learning. Morales et al. [31] employed transfer learning techniques to reuse kernels in convolutional neural networks across different domains. Maekawa et al. [28] trained a target user's activity model on labeled sensor data from source users with similar physical traits to those of the target user. Huynh et al. [11] used topic models to cluster activity data in an unsupervised manner.

Due to the recent growing interest in smart manufacturing and Industry 4.0 [7, 21, 34], studies on recognizing and supporting factory work using sensor technologies $[1,3-5,13]$ have been attracting attention. Of particular note are the previous studies done on monitoring and analyzing factory work using wearable sensors. One such study is Koskimäki et al. [16] in which they obtained acceleration and gyroscopic data from a wrist-worn inertial sensor device and analyzed operation processes in a line-production system to ensure that all necessary operations were performed. They recognized activities such as hammering and screwing using a $k \mathrm{NN}$ search. Another such study is Ward et al. [40] in which they obtained acceleration and audio data from a wrist-worn device to recognize woodworking activities by using HMMs and a linear discriminative classifier. Stiefmeier et al. [38] focused on assembly work on automobiles and used inertial sensors attached to several body parts, such as the upper and lower arms, to classify sensor data segments by computing the distance between the collected segments and sensor data templates prepared in advance. Examples of the activities used in their work include opening an engine hood and opening a trunk. Stiefmeier et al. [37] also studied the similar task of bicycle repair and used motion sensors and ultrasonic hand tracking to recognize maintenance activities using HMMs. All of these methods for analyzing factory work relied on supervised machine learning approaches and so required the creation of labeled training data.

The most relevant recent work is Maekawa et al. [27] in which they measured the duration of each work period on a production line in an unsupervised manner. Their method tracked a motif (sensor data segment) that appears only once in each work period using a particle filter to estimate the duration of the periods. Our work uses this method to roughly capture the structure of work periods during our preprocessing stage.

\section{UNSUPERVISED FACTORY ACTIVITY RECOGNITION METHOD}

\subsection{Assumed Environment}

3.1.1 Sensor Setting. Our method assumes that workers will be wearing a body-worn inertial sensor, i.e., a three-axis accelerometer. In our experiment, the workers wore a smart watch on their right wrists, with the smart watches' accelerometers collecting data at a sampling rate of approximately $60 \mathrm{~Hz}$.

3.1.2 Process Instructions. Process instructions are prepared in advance by a line manager for each work process to be done by a worker. They specify the flow of operations included in a work process, e.g., (1) place a board on the workbench, (2) check the test equipment mode, and (3) change the signal monitor mode, with some work processes containing optional operations, e.g., (4) pack accumulated boards into a box once 10 have been completed. These instructions also describe and specify the standard duration of each operation. For example, an operation named "preliminary preparation" had the following description: "Place the module in front of the body and fix it to the jig. Then connect a communication module and pick up the screwdriver." Note that this is translated from the original Japanese text used as input for our method. 


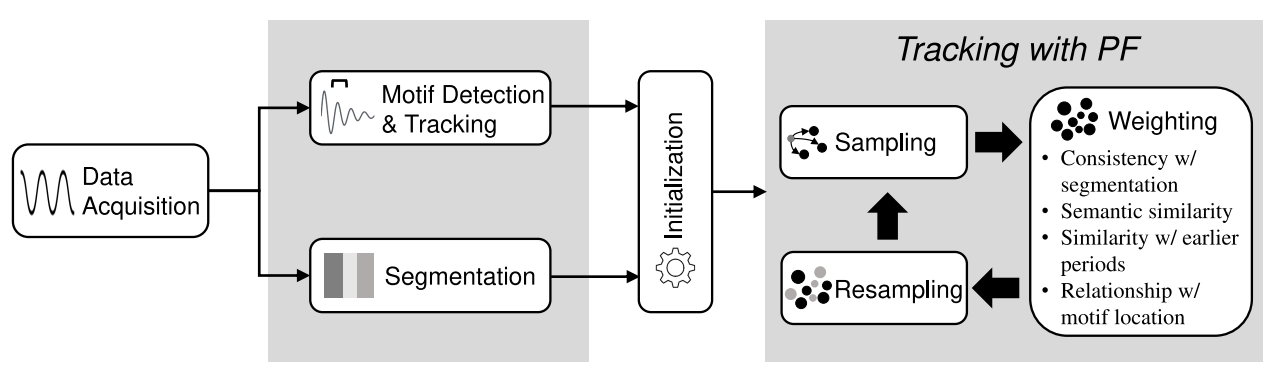

Fig. 2. Overview of the proposed method

\subsection{Overview}

Figure 2 shows an overview of the proposed method. We start with a time series of acceleration data that has been collected from a worker that corresponds to multiple iterations of that worker's work period. We then identify and track a motif that occurs once per work period in this sensor data. This motif is a unique data segment that occurs only once in each work period, e.g., the data corresponding to a hammering action that is performed only once in each work period. Since this motif occurs only once per work period, the period of sensor data spanning between the first and second occurrences of this motif must contain the starting time of the second work period, as shown in Figure 1.

In addition to the large-scale segmentation done using motifs, we also segment the sensor data on a smaller scale using a nonparametric Bayesian HMM. As can be seen in Figure 1, visible trend changes are evident at the transition points between many of the operations. Such trend changes can be detected using HMMs and can be considered as possible start times for individual operations in the work period, including the start time of the first operation in the work period, i.e., the start time of the overall work period.

Having used the motifs to identify the region of sensor data containing the start time for the second work period and used the trend changes to detect possible start times for each operation, we then run our particle filter. First we initialize our particle filter by generating an initial particle at a discovered trend change, representing a likely start point for the second work period (i.e., the start point of the work period's first operation). Then, as shown in Figure 3, particles are generated (sampled) along possible paths from the work period's start point through all the individual operation start times by iteratively sampling, weighting, and resampling at each estimated operation start time to generate estimates for the next.

The particle filter begins with the sampling phase, in which it uses a work model that encapsulates data derived from the process instructions to generate candidate start times (particles) for the following operation in the work period from an initial particle. In the weighting phase, the candidate start times (particles) are assigned weights based on (i) trend changes in the sensor data detected by HMM, (ii) semantic similarities between operations based on our work model, (iii) data similarities between operations and corresponding operations in preceding work periods, and (iv) the consistency of the motif's location in the current work period with its location in the previous work period. In the resampling phase, the particle filter resamples the candidate particles based on their weights, leaving it with the most likely start times for that operation. Each of these remaining particles then serve as initial particles when starting the sampling phase of the next iteration. At the end of each work period, we choose our estimated sequence of operation start times as being the path generated by our particle filter with the highest weight. 


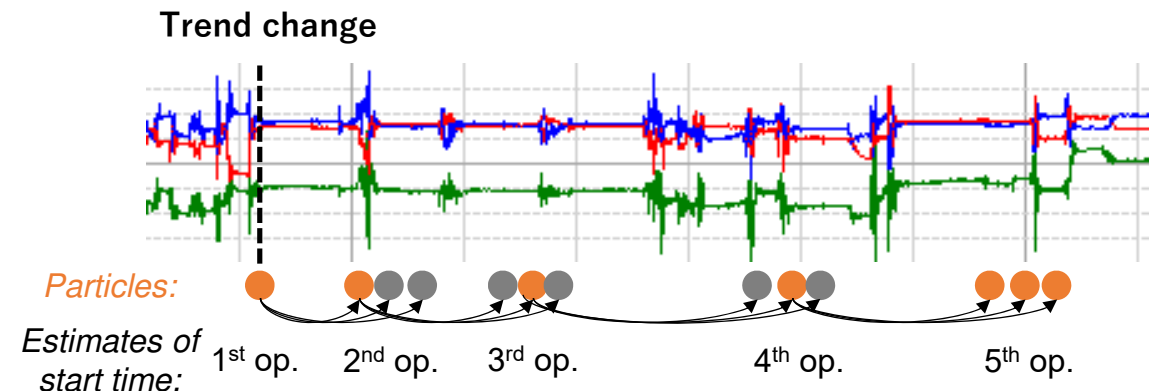

Fig. 3. An example of how paths of estimated start times for each operation are generated using a particle filter. We start with an initial particle at some trend change between the first and second motifs, which is a candidate start time for the first operation. We then sample several particles from this initial particle (three in this example), which are the initial estimates for the start time of the second operation. These sampled particles are then weighted and resampled, leaving us with our most likely estimates for the start time (one in this example). The most likely estimates are then sampled to generate the estimates for the start time of the third operation. The particle filter continues in this way, iteratively generating the start times for each operation based on its estimates for the start time of the previous operation.

\subsection{Work Model}

The first step in our process is to construct a work model that encapsulates the information obtained from the process instructions. It consists of two main parts: a tree-structured representation of the flow of operations and a matrix-structured representation of the semantic similarity between the operations' description texts. The flow of operations is represented as a tree structure whose root node corresponds to the first operation with at least one additional node in the tree for every possible operation in the work period, as shown in Figure 4. Edges connect each operation with the operations immediately preceding and following it, with branches possible in the case of optional operations, e.g., the branch before the optional "Boxing" operation in Figure 4. Each tree node contains information about the expected duration of the operation of interest, modeling this duration as a lognormal distribution. This operation flow model is then used to sample the durations of operations when generating particles to use as candidate start times for operations.

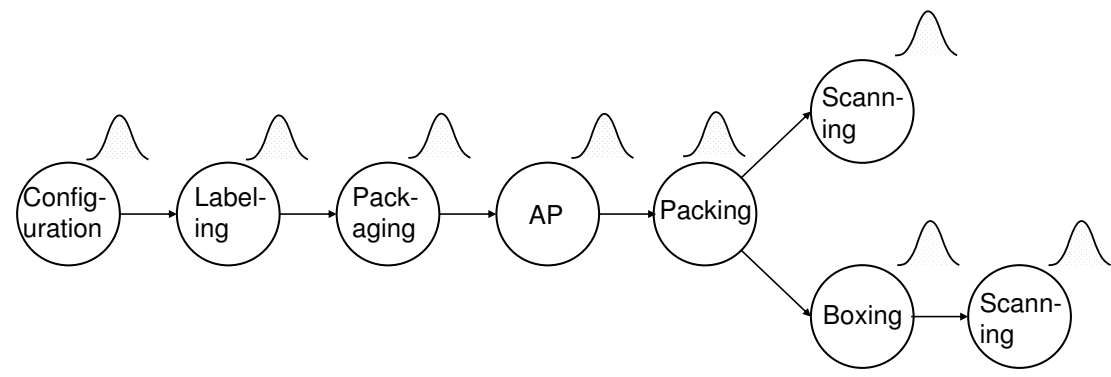

Fig. 4. Example flow of operations in the tree structure. (Due to the proprietary nature of these operations, only abstracted names are shown.)

The semantic similarity matrix allows us to estimate the sensor data similarity between operations based on the semantic similarity of their descriptions in the process instructions. Because our process instructions are 
written in Japanese, we first perform a morphological analysis of the descriptions based on [17], during which we tokenize the Japanese descriptions (i.e., perform text segmentation) and extract the verbs and nouns from the descriptions to use as keywords for the description. We capture the semantics of these keywords by generating a word vector for each keyword using a word2vec model [30] that is trained externally using the Japanese edition of Wikipedia ${ }^{1}$ to obtain a word vector that represents the semantics of that keyword. The keywords for each description are combined by computing the mean vector for all word vectors generated from that description, which is then regarded as the semantic vector for that description. Finally, we compute the cosine similarity between each pair of mean vectors to construct our semantic similarity matrix for the operations.

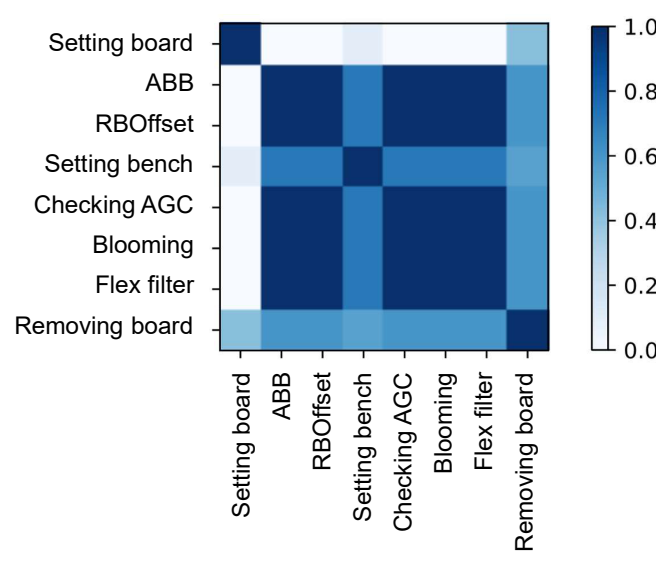

(a) Semantic similarities between operations
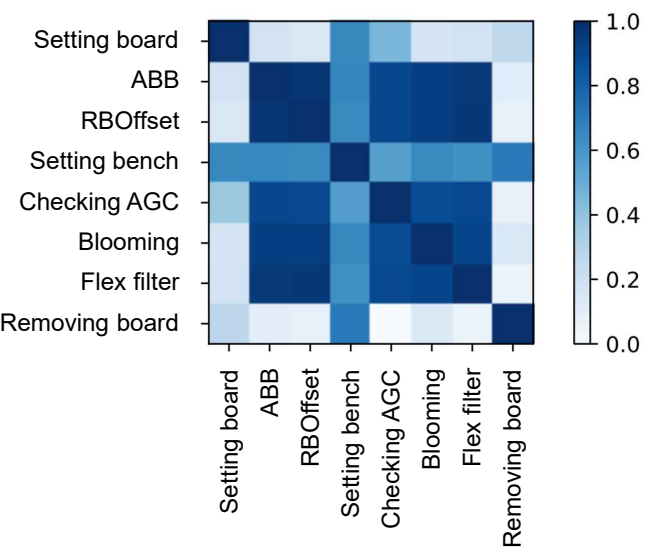

(b) Sensor data similarities between operations

Fig. 5. Example semantic similarity matrix and sensor data similarity matrix of a work period. (Due to the proprietary nature of these operations, only abstracted names are shown.)

Figure 5 (a) shows an example of a semantic similarity matrix for a work process. As is shown in the matrix, the semantic similarities between the "ABB," "RBOffset," "Checking AGC," "Blooming," and "Flex filter" operations are all high. All of these operations relate to manipulating dials when testing modules on a board. Figure 5 (b) shows a similarity matrix for the same work, with this matrix calculated using dynamic time warping [32] to compare ground truth sensor data segments of the operations. Here we can see the same overall trend in operation similarities as is seen in the semantic similarity matrix, with the dialing operations all showing relatively high similarity to each other. Therefore, the semantic similarity matrix can be used to evaluate each potential segmentation of a work period based on how well the sensor data similarities between segments match our expectations based on their semantic similarities.

\subsection{Motif Detection and Tracking}

The first step in identifying the start time for an overall work period is to identify and track a motif that occurs only once in each work period based on the method proposed in [27]. This method takes a time series of acceleration data and the standard duration of a work period from our work model as input and outputs timestamps for the locations of the most likely motif found in the sensor data (see Figure 1 for an example of this output). This motif corresponds to some characteristic action that is completed once per work period, e.g., a drilling action that is performed only once in each work period. Because the starting time of the $n$-th work period exists

\footnotetext{
${ }^{1}$ http://www.cl.ecei.tohoku.ac.jp/rm-suzuki/jawiki_vector/
} 


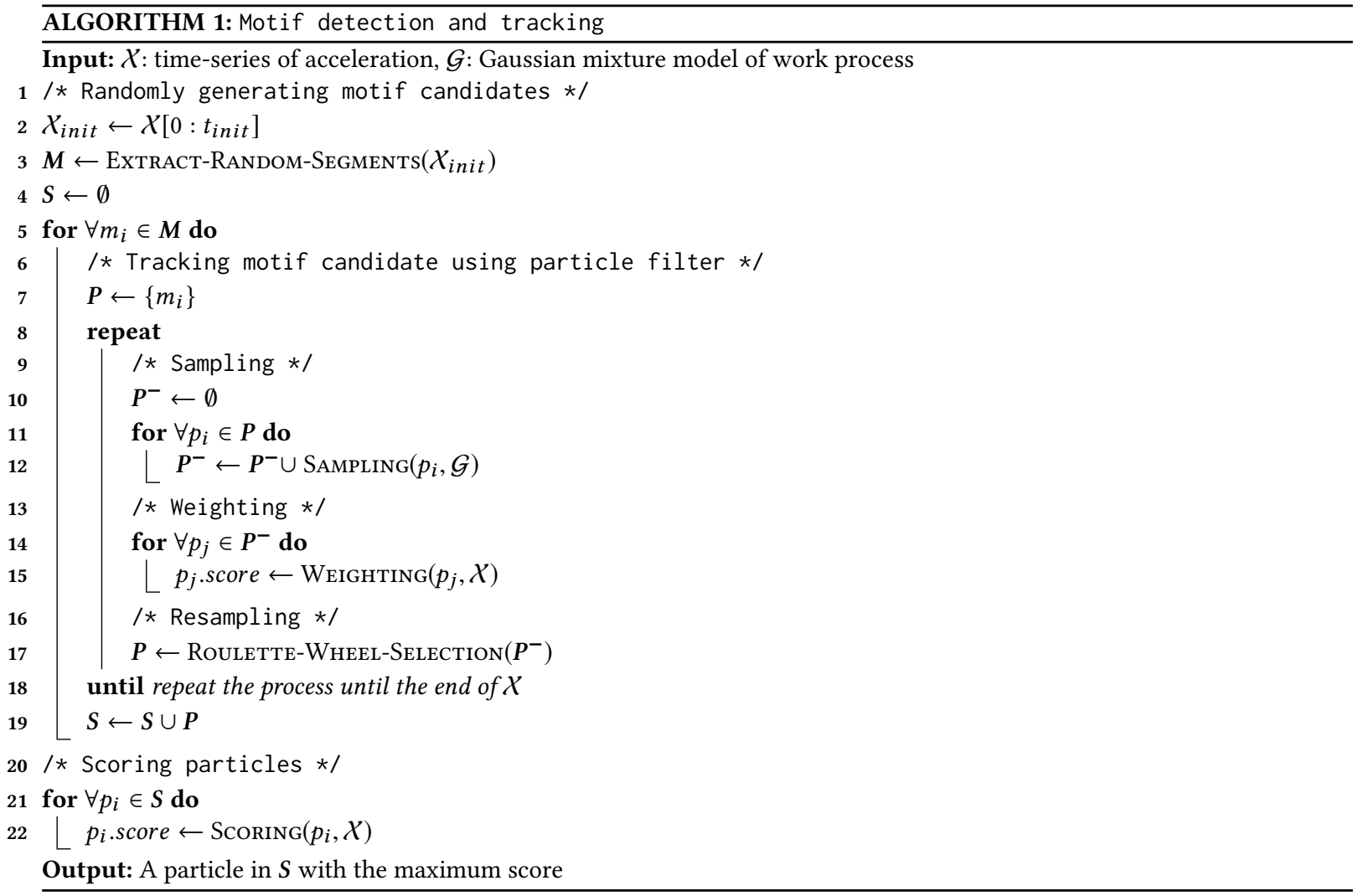

between the $n$-1-th and $n$-th occurrences of the motif, as shown in Figure 1, we can then narrow our search for this starting time to the area between these two occurrences of the motif. Algorithm 1 shows the algorithm used during motif detection.

3.4.1 Distribution of Durations for Work Periods. When searching for our motif, we need to model the duration (lead time) of the work period based on the work model we generated from the process instructions. We can model this lead time using a Gaussian mixture model (GMM), which is described as follows:

$$
p(t)=\sum_{i=1}^{N} \pi_{i} \mathcal{N}\left(t, \mu_{i}, \sigma_{i}\right),
$$

where $N$ is the number of mixtures, $\pi_{i}$ is the mixture weight of the $i$-th multivariate Gaussian distribution of the GMM (with $\pi_{i}=1 / N$ in our implementation), and $\mu_{i}$ and $\sigma_{i}$ are the mean and variance of the $i$-th Gaussian distribution, respectively. The number of mixtures $N$ represents the total number of possible patterns for the lead time (duration) in the work period. In the example shown in Figure 4, there are two possible patterns for the lead time because of the optional operation "Boxing." Therefore, $N$ corresponds to the number of leaf nodes in the process flow tree, with each of the $N$ distributions representing a complete path from the root node to one of the leaf nodes in the tree. The mean of the $i$-th distribution is then computed by summing the standard durations of all nodes from the root node to the the $i$-th leaf node in the tree. 
3.4.2 Random Generation of Motif Candidates. We begin our motif search by randomly extracting motif candidates, i.e., sensor data segments with length $w$, from the sensor data between time 0 and time $t_{\text {init }}$ (line 1 in Algorithm 1). Time $t_{\text {init }}$ is a hyperparameter for the algorithm, set as half of the standard lead time in our work. We then use a particle filter to generate the potential locations of subsequent occurrences for each candidate based on the lead-time distribution model described in Equation 1.

3.4.3 Tracking Motif Candidates Using a Particle Filter. Using the locations of the randomly generated motif candidates as our starting locations, we then search for potential locations where each of these motif candidates will reoccur in the data using a particle filter (line 6 in Algorithm 1). This particle filter initializes a particle at the exact location (timestamp) of each one of our randomly generated motif candidates, with each particle storing information about the motif candidate it represents, i.e., the sensor data segment for the motif. The particle filter then generates each motif's potential subsequent locations by iterating through phases of sampling, weighting, and resampling as follows.

Sampling: We randomly sample the subsequent occurrences of each particle based on the lead-time GMM $\mathcal{G}$ (line 9 in Algorithm 1). Assuming that $t\left(p_{i}, n\right)$ is the timestamp for a particle $p_{i}$ generated during the $n$-th iteration of sampling, then the timestamp for some new particle $p_{j}$ from among the $m$ particles generated from $p_{i}$ during the $n+1$-th iteration of sampling is determined using the following equation:

$$
t\left(p_{j}, n+1\right)=t\left(p_{i}, n\right)+\Delta t
$$

where $\Delta t$ is the estimated interval between occurrences of the motif candidate that is randomly sampled from the GMM $\mathcal{G}$. Each of these $m$ particles generated from $p_{i}$ are then our estimates for the possible $n+1$-th occurrences of the motif, with each new particle $p_{j}$ storing time $t\left(p_{i}, n\right)$ along with the times for each of the previous particles in its chain (one from each of the $n$ sampling iterations) as a history of the $n$ occurrences of the motif candidate (in addition to storing the sensor data segment from the initial motif candidate $p_{a}$ ).

Weighting: After having sampled new particles based on the GMM, we assign each new particle a score as follows (line 13 in Algorithm 1). In brief, each particle $p_{j}$ is assigned a score that is highest when the motif candidate $p_{a}$ occurs at $t\left(p_{j}, n+1\right)$ and does not occur at any other time between $t\left(p_{i}, n\right)+w$ and $t\left(p_{j}, n+1\right)$, exclusively. By doing so, we can identify motifs that appear only once in a work period. More specifically, when assigning a score to particle $p_{j}$, we compare the sensor data segment for $p_{a}$ (with length $w)$ to all windows with length $w$ in the interval $\left[t\left(p_{i}, n\right)+w, t\left(p_{j}, n+1\right)+w\right]$. This score is increased with increasing similarity of $\left[t\left(p_{j}, n+1\right), t\left(p_{j}, n+1\right)+w\right]$ to $\left[t\left(p_{a}, 1\right), t\left(p_{a}, 1\right)+w\right]$ and is decreased with increasing similarity of any segment of length $w$ in $\left[t\left(p_{i}, n\right)+w, t\left(p_{j}, n+1\right)\right]$ to $\left[t\left(p_{a}, 1\right), t\left(p_{a}, 1\right)+w\right]$. For more details on calculating the scores used during weighting, refer to [27].

Resampling: The sampled particles are probabilistically resampled using roulette wheel selection (line 16 in Algorithm 1). In roulette wheel selection, items are selected one at a time with each item randomly selected according to a probability that is associated with that item. In the case of our method, the probability of selecting a particle is set as proportional to the score assigned to the particle during the weighting phase. Using this method, we can probabilistically select high-score particles and discard the remaining particles.

These three procedures are iterated to produce new generations of particles until reaching the end of the input sensor data.

3.4.4 Computing Final Scores and Selecting the Best Motif. The final step in motif detection is to select the best candidate motif (line 20 in Algorithm 1). Using the steps outlined above, we now have several candidate motifs for each of which we have several sequences of particles that represent possible occurrences of that motif across our sensor data. We first compute a single score for each of these candidate motifs, which represents the sequence 


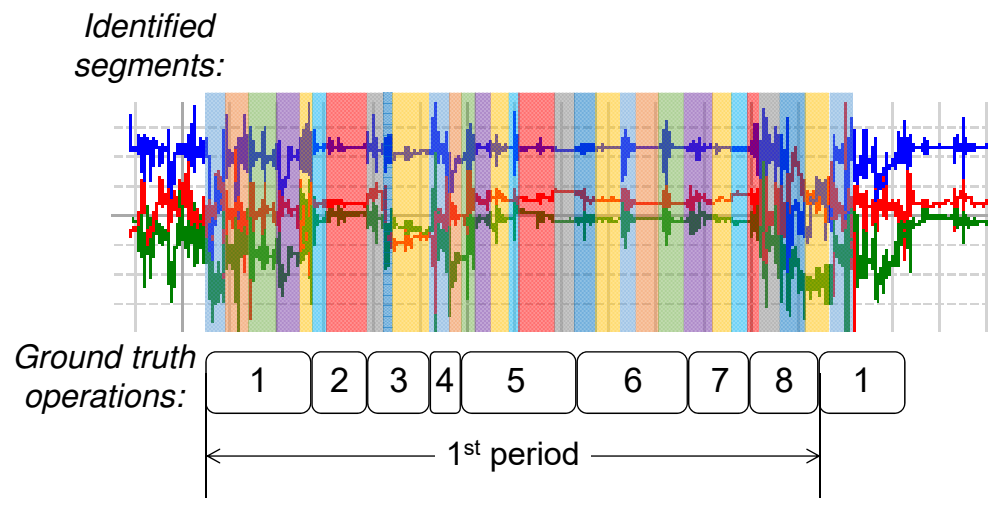

Fig. 6. Example segmentation result for acceleration data

of particles for that motif that had the highest weights. We then compare the candidate motifs based on these scores to select the best motif. For more details on motif scoring and selection, refer to [27].

\subsection{Segmentation}

In order to identify likely starting locations for work periods in the sensor data as well as likely starting locations for operations in the work periods, we segment the sensor data based on trend changes in the data. We do this in an unsupervised manner by employing a Bayesian nonparametric version of HMM called the hierarchical Dirichlet process HMM (HDP-HMM) [12]. HDP-HMM is a nonparametric model that allows the number of states to be learned from the data. The HDP is used to infer a posterior distribution of the number of states of an HMM, permitting us to decode (segment) a time-series using an HMM without manually specifying the number of states of that HMM. For more details about HDP-HMM, refer to [12].

Figure 6 shows an example result from this segmentation method when applied to acceleration data. The overlaid color rectangles represent the segments generated by HDP-HMM, while the numbered rectangles below the data show the ground truth operations for the data. As can be seen in this figure, while HDP-HMM segmentation resulted in many false positives, many of the ground truth segmentation boundaries were included in its results. Therefore, we can use HDP-HMM to generate a list of candidate start times for the individual operations, which in turn can also be treated as candidate start times for the work period.

\subsection{Tracking with Particle Filtering}

3.6.1 Overview. Using the methods described in Section 3.4 and Section 3.5, we are able to obtain timestamps for the occurrences of a motif $\mathbf{M}$ and timestamps for trend changes (candidate start times) $\mathbf{C}$. We now want to use a particle filter to produce our estimate for the most likely starting time for our work period along with the starting times for all operations in that work period. We start by initializing this particle filter based on $\mathrm{C}$ and $\mathbf{M}$ to generate particles that act as candidates for the starting time of the work period. We then track each of these particles to identify the starting times of the other operations in the work period by iterating through the particle filter's three phases of sampling, weighting, and resampling as shown in Figure 3. Figure 7 illustrates the sampling, weighting, and resampling procedures, while Algorithm 2 shows the algorithm of this procedure.

3.6.2 Initialization. As can be seen in Figure 1, the starting time of the second work period exists somewhere between the first and second occurrences of our motif. Therefore, we can generate initial particles at each timestamp from $\mathrm{C}$ that fall between the first and second occurrences of the motif (found in $\mathrm{M}$ ), which become our 


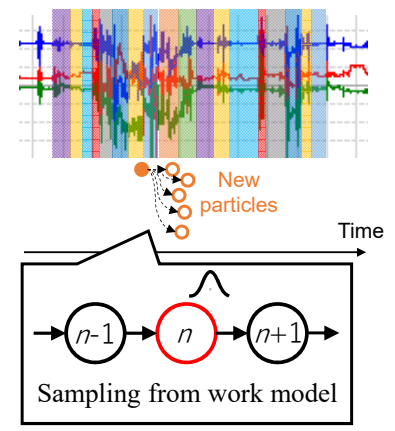

(a) Sampling for $n$-th operation

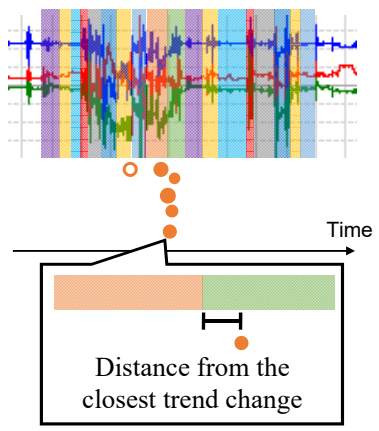

(b) Weighting for $n$-th operation

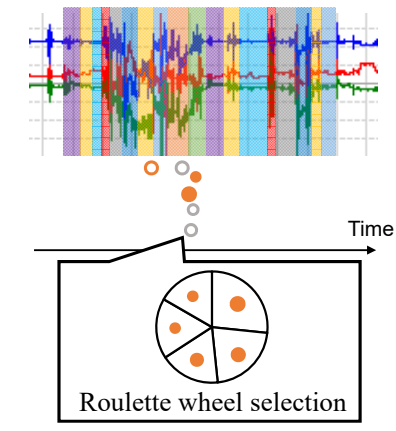

(d) Resampling for $n$-th operation

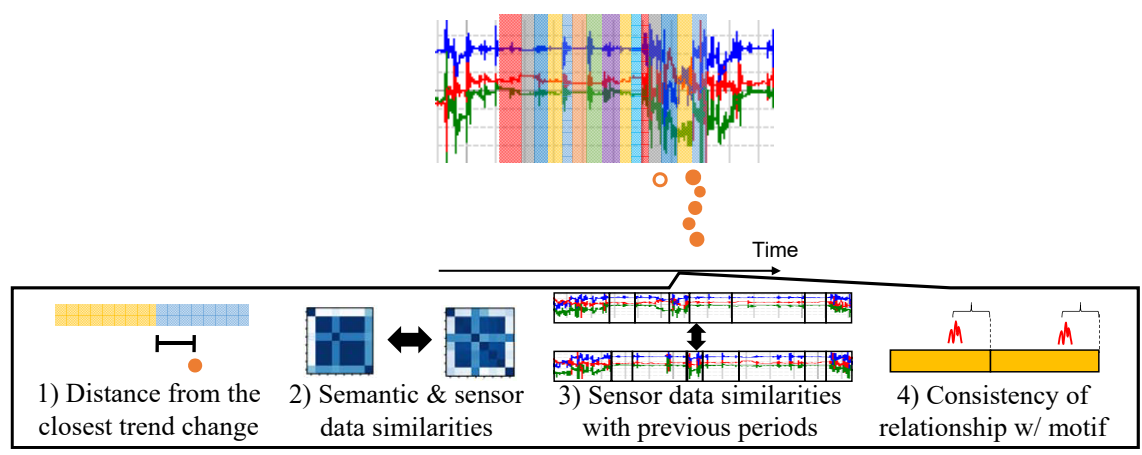

(c) Weighting for the last operation

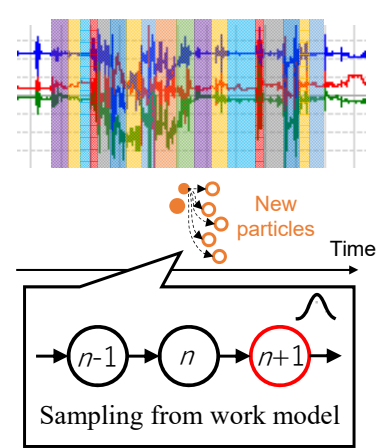

(e) Sampling for $n+1$-th operation

Fig. 7. Sampling, weighting, and resampling procedures in the proposed method

candidates for the starting time of the first operation in the second work period (line 1 in Algorithm 2). We also set the current state of each initial particle as the root node (first operation) of the operation flow from our work model.

3.6.3 Sampling. As shown in Figure 7 (a), with each iteration we sample new particles from each existing particle that represent estimates of the starting time for the following operation (line 17 in Algorithm 2). Assuming that $t\left(p_{i}, n, k\right)$ is the timestamp for an existing particle $p_{i}$ that represents the starting time of the $n$-th operation in the $k$-th work period, then the timestamp $t\left(p_{j}, n+1, k\right)$ for a new particle $p_{j}$ for the $n+1$-th operation is sampled from $p_{i}$ as follows:

$$
t\left(p_{j}, n+1, k\right)=t\left(p_{i}, n, k\right)+\Delta t,
$$

where $\Delta t$ is randomly sampled based on the duration-time distribution found in the currently selected node in the work model's operation flow (i.e., the $n$-th operation's node). Therefore, $t\left(p_{j}, n+1, k\right)$ is an estimate of the starting time of the $n+1$-th operation. Note that each new particle $p_{j}$ stores time $t\left(p_{i}, n, k\right)$ along with the times of all previous particles in its chain as a history of the starting times for previous operations.

3.6.4 Weighting. Each particle generated in the sampling phase is then assigned a weight using a combination of four scores: a trend-based score, a semantics-based score, a sensor-based score, and a motif-based score (line 22 in Algorithm 2). We determine which of these scores to use depending on the current state of the particle, 


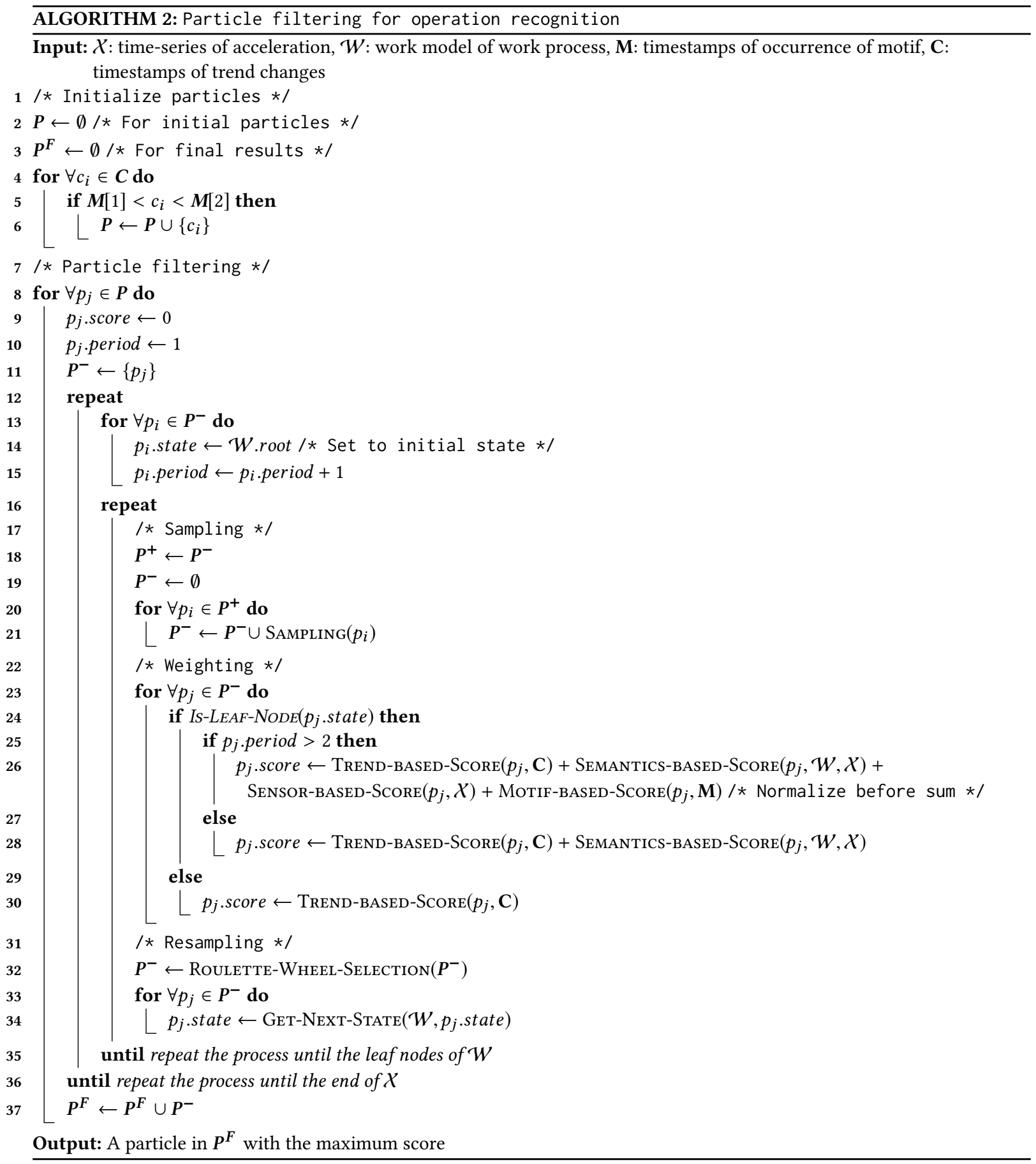


with the trend-based score always used, as shown in Figure 7 (b), and the semantics-based, sensor-based, and motif-based scores used when the state corresponds to the final operation of a work period, as shown in Figure 7 (c). These scores are calculated as follows:

Trend-based score: This score enables us to detect operation starting times based on the trend changes discovered by HDP-HMM. As shown in Figure 7 (b), this score is computed based on the temporal distance between the timestamp of a particle and the timestamp of the closest trend change in $\mathrm{C}$ as follows:

$$
\left.\operatorname{CumulativeDist}\left(p_{j}, n, k, \mathbf{C}\right)=\sum_{m=2}^{n+1} \operatorname{Dist}\left(t\left(p_{j}, m, k\right), \mathbf{C}\right)\right)
$$

where $t\left(p_{j}, m, k\right)$ is the timestamp for the ancestor particle of $p_{j}$ corresponding to the $m$-th operation in the $k$-th work period (when $m$ is equal to $n+1$, it is the timestamp for $p_{j}$ itself) and

$$
\operatorname{DisT}(t, \mathbf{C})=\min _{c \in \mathbf{C}}|t-c| \text {. }
$$

The inverse of this distance is then used as the score, with this score used each time we compute weights for particles.

Semantics-based score: Unlike the trend-based score that can be computed for each new particle, the semantics-based score can only be computed for particles representing the end of a work period, i.e., the end time of the final operation. This score enables us to compare two operations in a work period to see how well the similarities in sensor data for the two operations match with the semantic similarities in the descriptions for those operations. As mentioned in Section 3.3, we calculate the semantic similarity between each pair of descriptions for operations to form the semantic similarity matrix. We then concatenate all entries below the main diagonal in the matrix to form a semantic-similarity vector. As mentioned in Section 3.3, we also calculate a sensor-data similarity matrix for all pairs of operations, with the sensor-data for each operation extracted based on the timestamps of $p_{j}$ and all of its ancestor particles leading back to the first operation $(n=1)$ in the same period. Again we concatenate all entries below the main diagonal to form a sensor-data similarity vector. Finally, we compute the cosine similarity between the semantic-similarity vector and the sensor-data similarity vector as the semantics-based score. Note that, when optional operations are not observed in the work period, we ignore them during the similarity calculation.

Sensor-based score: Like the semantics-based score, the sensor-based score is only computed for particles representing the end of a work period. It enables us to compare two work periods by leveraging the expected similarity of sensor data for the same operation even when conducted in different work periods. We calculate the sensor data similarity for each operation using dynamic time warping (DTW) as follows:

$$
\operatorname{DTW}\left(\mathcal{X}\left(p_{j}, n, k\right), \mathcal{X}\left(p_{j}, n, k-1\right)\right),
$$

where $D T W(\cdot, \cdot)$ calculates the DTW distance between two sensor data segments. Here, $\mathcal{X}\left(p_{j}, n, k\right)$ gives the sensor data segment of the $n$-th operation in the $k$-th work period identified by particle $p_{j} . \mathcal{X}\left(p_{j}, n, k-1\right)$ gives the sensor data segment of the $n$-th operation in the $k$-1-th work period identified by an ancestor particle of $p_{j}$ corresponding to the $n$-th operation in the $k$-1-th work period. We compute the sum of the dynamic time warping distances and the regard the inverted value as the sensor-based score. Note that, to reduce computation costs, we standardize and then discretize the two sensor data segments, and then represent the segments as piece-wise approximations where the time-series is divided into equal-sized frames and the mean value of data within each frame becomes a representative value of the frame [19].

Motif-based score: Like the semantics- and sensor-based scores, the motif-based score is only computed for particles representing the end of a work period. Because the motif tracking method [27] finds motifs 
that occur only once per work period, motifs will occur with an interval corresponding to the lead time for the work period, with the location of a motif within any work period consistent across adjacent work periods (see Figure 1), even when some work periods contain optional operations (which is proven in [27]). Therefore, we can evaluate the quality of an estimated series of operation start times based on the consistency of motif locations in the estimated work periods. We start by computing two values for each occurrence of a motif that measure the temporal distances from the motif to the start time $\left(d_{s}\right)$ and end time $\left(d_{e}\right)$ of the work period. We then use these temporal distances to compare the $k$-th and $k-1$-th occurrences of a motif by computing the absolute difference between each value for $k$-th and $k$ - 1 -th occurrences (e.g., $\left.\left|d_{s}^{k}-d_{s}^{k-1}\right|\right)$, using the smaller of these two absolute differences in the motif-based score.

Additionally, since the motif should be observed in sensor data segments corresponding to the same operation in each work period (as shown in Figure 1), we also compute the temporal distance between its location and the starting/ending time of the operation in which it occurs. We then incorporate the absolute difference between these distances for the current work period and the previous work period as part of the motif-based score. Furthermore, when the motif does not appear in the same operation for the current and previous work periods, we apply a penalty to the score. This score enables us to reduce the overall score for estimated paths that do not match the standard lead time of the work period.

The four scores described above are computed at the end of each work period. The final score used for any given particle is then calculated as the cumulative sum of these four scores for the current particle together with these scores from all of its ancestor particles (i.e., the particles in its path from each previous work period).

3.6.5 Resampling. The sampled particles are probabilistically resampled using roulette wheel selection as shown in Figure 7 (d) (line 31 in Algorithm 2). We then set the current state for each remaining particle as the following operation in the operation flow (line 34 in Algorithm 2). In the case of a branch in the operation flow, we randomly select the branch to follow. Each of these remaining particles is then used to generate new particles during the sampling phase of the next iteration, as shown in Figure 7 (e). Note that, because we can only calculate the trend-based and semantics-based scores at the end of the second work period, the weighting used at this point yields less precise estimates of operation start times than are possible with later work periods. Therefore, at the end of the second work period, we resample two times as many particles as are sampled for later periods. In addition, because the durations of optional operations such as "boxing accumulated items" and "waiting on an item from the previous worker" often have high variance, we also resample two times as many particles for these operations as the other operations. Further, because an estimate of the ending time of the last operation of the $k$-th work period should exist between the $k$-th and $k+1$-th occurrences of the motif as shown in Figure 1 , we discard estimates (particles) for the ending time of the last operation that do not satisfy this condition.

We iterate through sampling, weighting, and resampling until reaching the end of the sensor data, at which point we select the particle with the highest weight as representing the most likely sequence of start times for operations in the sensor data.

\section{EVALUATION}

\subsection{Dataset}

We evaluated the proposed method using data collected from factory workers in actual factories. Table 1 gives an overview of the work processes observed for this study. The "\# operations" row in the table shows the number of standard operations included in each work process, with the number of optional operations shown in parentheses. For example, work process A consists of 11 standard operations along with one optional operation. The "standard duration" row shows the standard duration of one work period for each work process, with the standard duration when optional operations are performed shown in parentheses. For example, the standard 
Table 1. Overview of our dataset

\begin{tabular}{|c||c|c|c|c|c|c|}
\hline & work A & B & C & D & E & F \\
\hline \hline \# operations & $11(1)$ & 8 & $6(2)$ & 11 & 7 & $7(1)$ \\
\hline standard duration [s] & $50(59)$ & 124 & $55(115)$ & 52 & 55 & $46(60)$ \\
\hline periods & 11 & 12 & 10 & 12 & 6 & 7 \\
\hline data duration [s] & 655 & 1440 & 614 & 639 & 360 & 420 \\
\hline work overview & $\begin{array}{c}\text { install screws } \\
\text { on circuit } \\
\text { board }\end{array}$ & $\begin{array}{c}\text { test and record } \\
\text { circuit board } \\
\text { information }\end{array}$ & $\begin{array}{c}\text { bag and box } \\
\text { circuit } \\
\text { boards }\end{array}$ & $\begin{array}{c}\text { install screws } \\
\text { on circuit } \\
\text { board }\end{array}$ & $\begin{array}{c}\text { check final } \\
\text { product and } \\
\text { record results }\end{array}$ & $\begin{array}{c}\text { check final } \\
\text { product and } \\
\text { record results }\end{array}$ \\
\hline
\end{tabular}

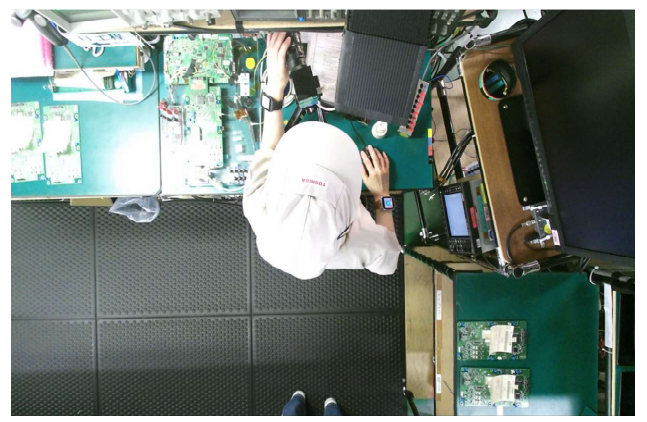

Fig. 8. Image from video captured in the factory

duration of work process $\mathrm{A}$ is $50 \mathrm{~s}$ without the optional operation and $59 \mathrm{~s}$ with the optional operation. The "periods" row shows the number of work periods included in the observed sensor data for each work process. The "data duration" row shows the total duration of collected sensor data for each work process. The standard duration of each operation was roughly determined by the actual duration of the operation; compute the average duration without outliers and keep the first decimal place.

The sensor data was collected using a Sony SmartWatch3 SWR50 attached to each worker's right wrist, with the acceleration data sampled at approximately $60 \mathrm{~Hz}$. Video was also recorded in order to obtain the ground truth information needed for evaluation, with Figure 8 showing an example of the images captured. All data processing was conducted offline in our laboratory after collection.

\subsection{Evaluation Methodology}

Our method provides the starting (and ending) times for all operations in each work period, allowing us to classify each sensor data point as belonging to an operation class (excluding data points in the first work period). During evaluation, we can then treat our results as classification results estimated per data point (acceleration sample), allowing us to calculate the macro-averaged F-measure of our results.

To evaluate the effectiveness of the proposed method, we prepared the following methods.

- Proposed: This is the proposed method.

- w/o Sem: The proposed method without the use of the semantics-based score during the weighting phase of the particle filter. Therefore, we assume a condition where description texts about operations are unavailable. 


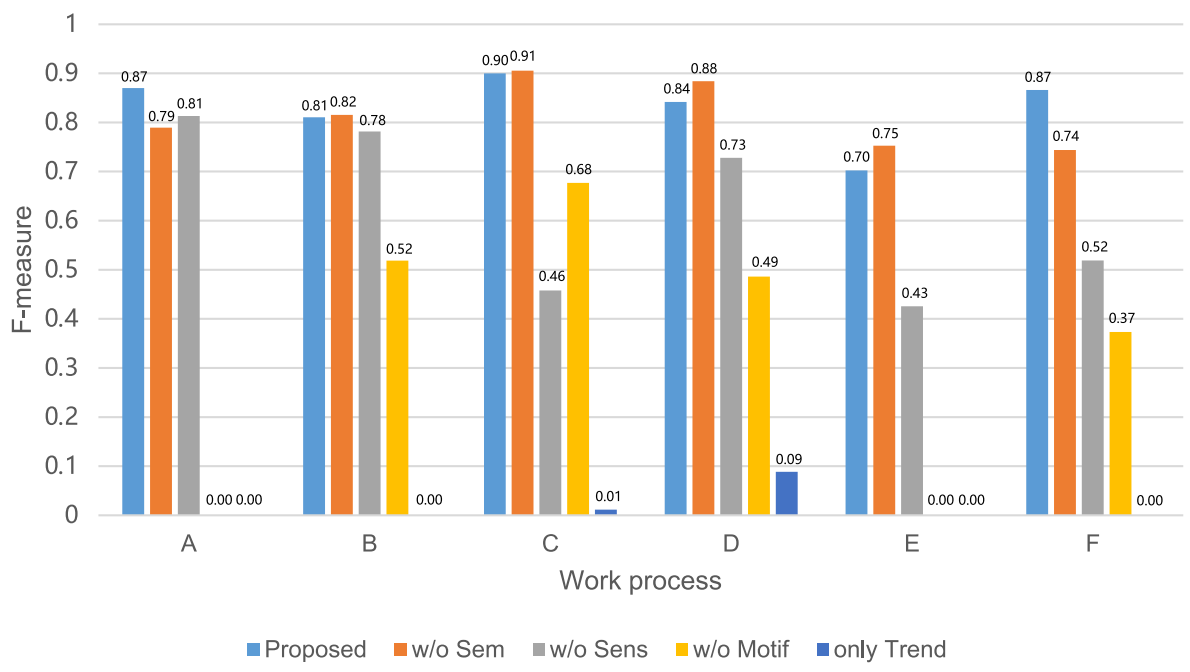

Fig. 9. Accuracies (F-measures) of the five methods calculated using the path of the particle with the highest score at the end of the final work period

- w/o Sens: The proposed method without the use of the sensor-based score during the weighting phase of the particle filter.

- w/o Motif: The proposed method without the use of the motif-based score during the weighting phase of the particle filter.

- only Trend: The proposed method using only the trend-based score during the weighting phase of the particle filter. That is, this method uses neither the semantics-based score, the sensor-based score, nor the motif-based score.

Here we explain the way of selecting hyper-parameters of HDP-HMM. The maximum number of HMM states is set as the number of operations. The hyper-parameter of the prior distribution on state-transition parameters, which relates to the duration of an operation, is set as the data length divided by ten plus a bias.

\subsection{Results}

4.3.1 Recognition Accuracy. Figure 9 shows the classification accuracy for each method. Note that because the methods used in this study are based on particle filters, each method can produce several estimated segmentation paths per work period. The results shown in Figure 9 select a single path when calculating the F-measure by selecting the path corresponding to the particle with the highest score at the end of the final work period. Additionally, the F-measures for the w/o Motif and only Trend methods are zero for some work processes because all particles were discarded in the resampling phase at the end of a work period, i.e., no particles satisfied the condition that estimates for the ending time of the last operation of the $k$-th work period should be between the $k$-th and $k+1$-th occurrences of the motif.

Overall, the Proposed method achieved the highest average F-measure across all six work processes (83.2\%), even though it employs an unsupervised approach. However, Proposed did not achieve the highest F-measure for all six work processes, with the F-measure of w/o Sem higher than that of Proposed for several work processes, despite performing slightly worse than Proposed overall (81.5\%). Also of note are the poor results from the only 


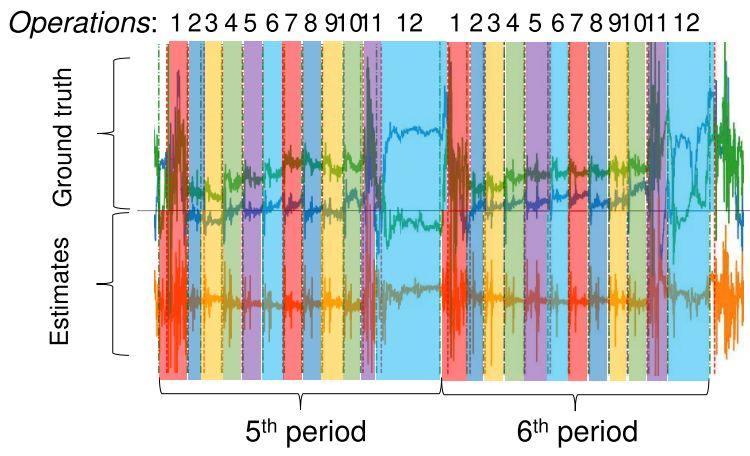

Fig. 10. Example output from Proposed for the 5th and 6th periods of work process A

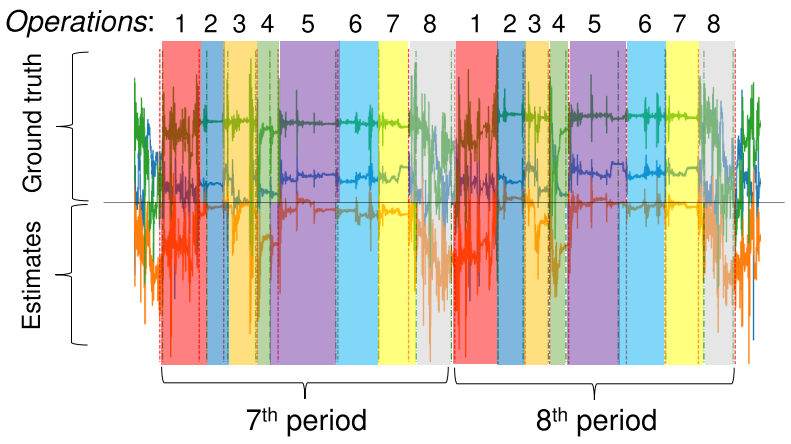

Fig. 11. Example output from Proposed for the 7th and 8th periods of work process B

Trend method, which indicate that it is not possible to recognize the operations using only trend changes in the sensor data.

Figure 10 shows an example output from the Proposed method for the fifth and sixth periods of work process A. The individual operations are indicated by the colored bars, with the upper half of the bars indicating the ground truth segmentation and the lower half of the bars indicating the estimated segmentation output by Proposed. The numbers in the upper margin correspond to the operation number for each ground-truth segment. From this figure, we can see that the estimates from Proposed match closely with the ground truths and that Proposed was even able to identify the optional operation (operation 12) found at the end of each period with only small errors in the ending and starting times of the neighboring operations (operations 11 and 1). Additionally, since the Proposed method leverages the correlation between semantic and sensor-data similarities, it was able to do well at identifying operations 2 to 10 , which all correspond to screwing operations with high semantic and sensor-data similarities.

Figure 11 shows another example result from the Proposed method. In this case, the operations in the work process do not have similar sensor data like in work process A, but Proposed is still able to accurately identify the operations with only small errors by leveraging the trend-based, sensor-based, and motif-based scores.

Figure 12 shows an example output of Proposed for work process E, for which the F-measure of Proposed is somewhat poorer than for the other work processes. This may be due to the large variance in the durations of the operations in work process E, as can be seen when comparing the consecutive segments for operations 


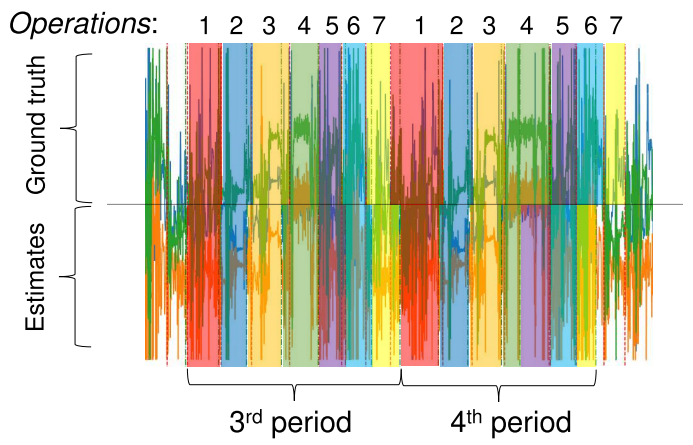

Fig. 12. Example output from Proposed for the 3th and 4th periods of work process $E$

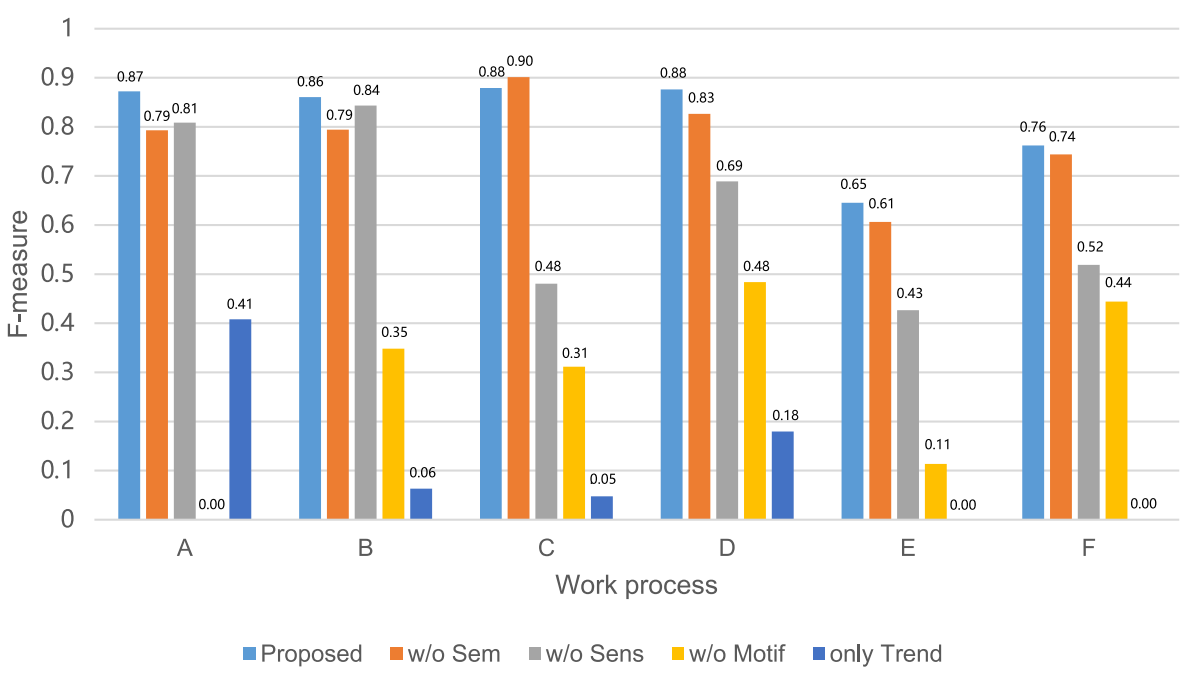

Fig. 13. Accuracies (F-measure) of the five methods calculated from the path of the particle with the highest score at the end of each work period

1 or 4 . Because this work process involves inspecting items, the duration of some operations will depend on condition of the items.

Figure 13 shows the average F-measure per work period for each method, with the path used for each work period selected as the path with the highest score at the end of that work period. This differs from Figure 9 in which the results are based on a single contiguous path across all work periods. Interestingly, the F-measure of Proposed is higher than that of w/o Sem and w/o Sens for many work processes in this figure. Comparing Figure 13's per-work-period averages to Figure 9's per-work-process averages, we see that when we use fewer scores (i.e., w/o Sem and w/o Sens), we can still achieve high accuracy when we can consider the entire work process's sensor data when generating the estimates. In contrast, when we can use only the sensor data up to and including the work period of interest, using all the scores (i.e., Proposed) provides the most accurate estimates. Additionally, the higher F-measures in Figure 9 when compared to Figure 13 indicate that these methods could improve on their estimates from past work periods when processing an entire work process. This ability to 


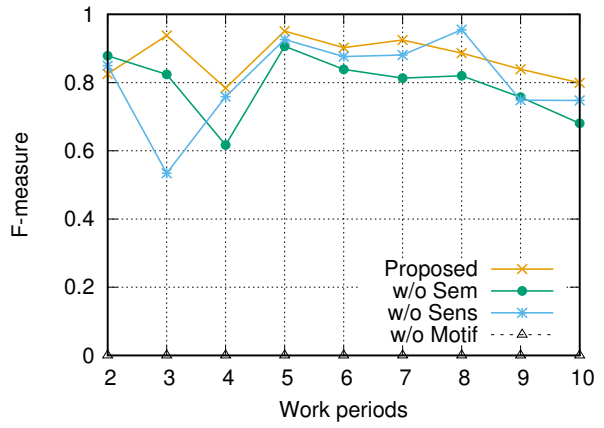

Results from work process A

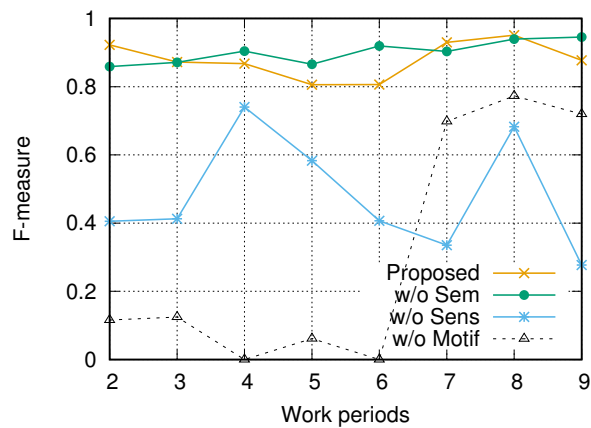

Results from work process C

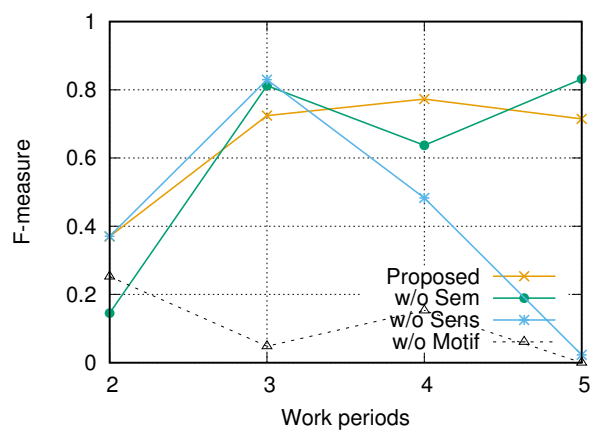

Results from work process $\mathrm{E}$

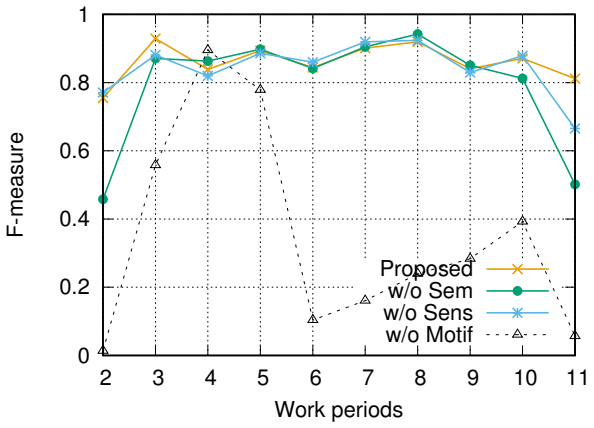

Results from work process B

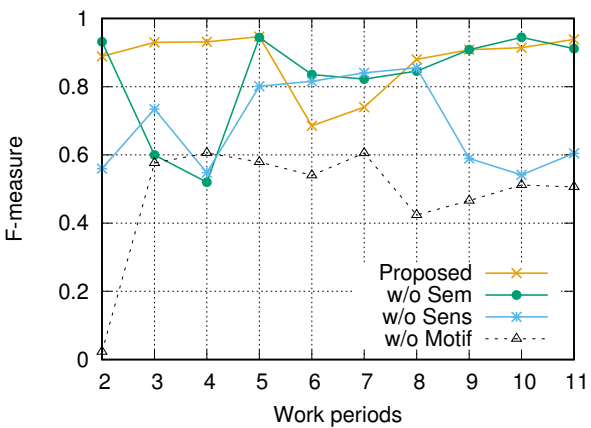

Results from work process D

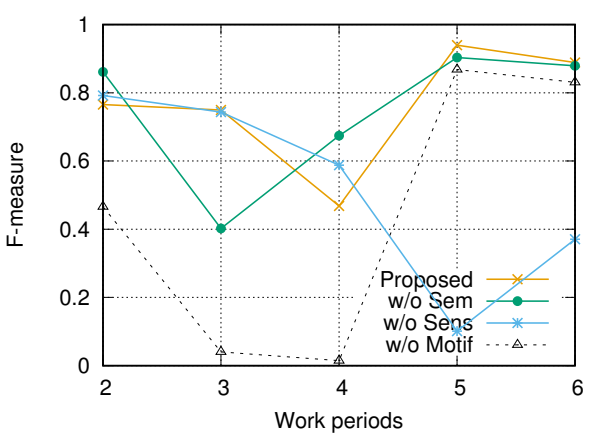

Results from work process $\mathrm{F}$

Fig. 14. Transitions in the accuracies (F-measure) of the methods calculated from the path of the particle with the highest score at the end of each work period

improve on earlier estimates comes from how the timing of each work period is dependent on the previous work period, meaning that a contiguous path that does well at predicting the timing of given work period is likely to have done well at predicting at the very least the ending of the previous work period.

4.3.2 Transitions in Recognition Accuracy. Figure 14 breaks down the average F-measures from Figure 13 into the individual F-measures for each work period. Here we can see that the F-measure for the second work periods 


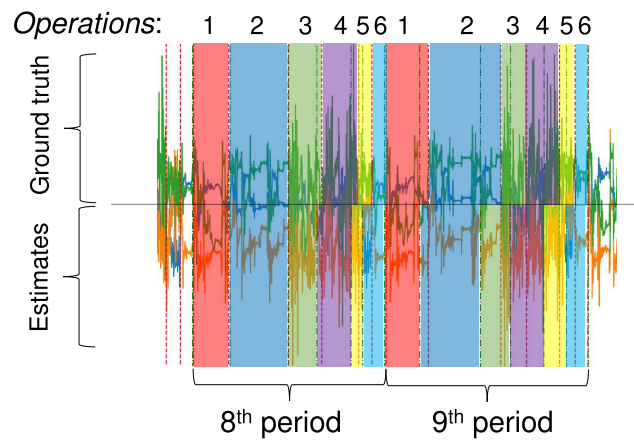

Fig. 15. Example output from w/o Sens for the 8th and 9th periods of work process $C$

seems to be poor for many work processes. This is because we calculate the particle scores using only the trendand semantics-based scores at the end of the second work period, while later work periods benefit from including the sensor- and motif-based scores.

In addition, we can find sudden decreases in the F-measure. This is mainly caused by the first occurrence of an optional operation. Because we cannot employ the sensor-based score for such operations, the F-measure significantly decreases in such cases. Further, as can be seen in the results, the transitions of Proposed seem to be more stable than those of the other methods. This robust estimation is achieved by increasing the diversity of input by incorporating all four score functions.

4.3.3 Effect of Semantic Similarity. As can be seen in the w/o Sem results in Figures 9 and 13, the F-measure of w/o Sem is higher for many work processes than those of w/o Sens and w/o Motif. This indicates that the contribution of the semantics-based score is lower than the contributions of the sensor-based and motif-based scores. Although a semantic similarity matrix can often capture the overall trends in a work process, as shown in Figure 5, there is no guarantee that the semantic similarities are equivalent to the sensor data similarities. In particular, the F-measure of Proposed for process C is poorer than that of w/o Sem in Figure 13. In this case, the nouns used in the process instructions of many operations in work process $C$ were similar with each other despite the actions performed during the operations not being similar, resulting in a semantic similarity matrix that did not match well with the sensor data similarity matrix.

4.3.4 Effect of Sensor Data Similarity between Different Periods. Figures 9 and 13 also show the results for w/o Sens, which does not employ the sensor-based score. As can be seen in these results, the F-measures of w/o Sens are poor for many work processes, indicating that the sensor-based score was an important aspect of the Proposed method. This is because the sensor-based score increases the consistency of estimated start times across work periods by comparing the sensor data for each operation from the current and previous periods. For example, Figure 15 shows an example result for w/o Sens for work process $\mathrm{C}$, which was generated from the path of the particle with the highest score at the end of the final work period. As shown in this result, the operations identified by w/o Sens in consecutive periods are inconsistent. For example, segments of the second operation in the eighth and ninth periods identified by w/o Sens are not consistent, reducing the classification accuracy for later operations.

4.3.5 Effect of Relative Location of Motif. Looking at the w/o Motif results in Figures 9 and 13, we can also confirm the effectiveness of the motif-based score, since the F-measure of w/o Motif is poor for many work processes. The motif-based score ensures a consistent identification of the start times for the first operation and 


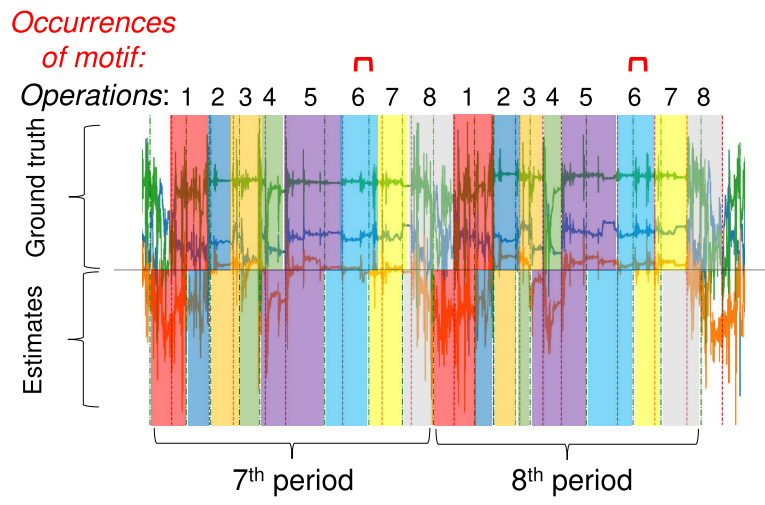

Fig. 16. Example output from w/o Motif for the 7th and 8th periods of work process B

the operation containing the motif. Figure 16 shows an example result for w/o Motif for work process B, in which the motif used by our method was located in the sixth operation. Because w/o Motif does not consider the location of the motif, the segments identified as the sixth operation in the two periods are inconsistent, with the motif included in the sixth operation of the seventh period and in the seventh operation of the eighth period.

As above, we could confirm the effectiveness of our particle filter-based architecture and our proposed scoring methods using sensor data collected in actual factories. We also confirmed that the motif-based score and sensorbased score are necessary to achieve high recognition accuracy. In addition, we confirmed that even when text descriptions of the operations are unavailable, the proposed method (w/o Sem) can still achieve high recognition accuracy.

\section{CONCLUSION}

This paper presented an unsupervised method for recognizing assembly work done by factory workers using wearable sensor data. The proposed method detects the starting and ending times of each operation included in a work process by leveraging information about (i) trend changes in the sensor data detected by HMM, (ii) semantic similarities between operations extracted from process instructions, (iii) data similarities between operations and corresponding operations in preceding work periods, and (iv) the consistency of a motif's location in the current work period with its location in the previous work period. We confirmed the effectiveness of the proposed method using sensor data collected from workers in actual factories. The Proposed method achieved a high average accuracy (F-measure) of about $80 \%$.

As a part of our future work, we plan to improve our method in order to cope with unexpected events that may arise in a factory. For example, the workers may unexpectedly halt their work when a problem occurs in the line production system. The sensor data used in this paper did not contain such unexpected events. In the future, we plan to automatically detect such non-work periods and stop the particle filtering process during these periods.

\section{ACKNOWLEDGMENTS}

This work is partially supported by JST CREST JP-MJCR15E2, JSPS KAKENHI Grant Number JP16H06539 and JP17H04679. The first author is supported by China Scholarship Council. 


\section{REFERENCES}

[1] Mario Aehnelt and Sebastian Bader. 2015. Information assistance for smart assembly stations. In the 7th International Conference on Agents and Artificial Intelligence (ICAART 2015), Vol. 2. 143-150.

[2] Mario Aehnelt, Enrico Gutzeit, and Bodo Urban. 2014. Using activity recognition for the tracking of assembly processes: Challenges and requirements. In WOAR 2014. 12-21.

[3] Mario Aehnelt and Bodo Urban. 2015. The knowledge gap: providing situation-aware information assistance on the shop floor. In $H C I$ in Business. 232-243.

[4] Mario Aehnelt and Karoline Wegner. 2015. Learn but work!: towards self-directed learning at mobile assembly workplaces. In the 15th International Conference on Knowledge Technologies and Data-driven Business. 17.

[5] Sebastian Bader, Frank Krüger, and Thomas Kirste. 2015. Computational causal behaviour models for assisted manufacturing. In the 2nd international Workshop on Sensor-based Activity Recognition and Interaction. 14.

[6] Ling Bao and Stephen S Intille. 2004. Activity recognition from user-annotated acceleration data. In Pervasive 2004. 1-17.

[7] Martin Bauer, Lamine Jendoubi, and Oliver Siemoneit. 2004. Smart Factory-Mobile Computing in Production Environments. In the MobiSys 2004 Workshop on Applications of Mobile Embedded Systems (WAMES 2004).

[8] Mark Blum, Alex Sandy Pentland, and Gehrard Tröster. 2006. Insense: Interest-based life logging. IEEE Multimedia 13, 4 (2006), 40-48.

[9] Jianfeng Chen, Alvin Harvey Kam, Jianmin Zhang, Ning Liu, and Louis Shue. 2005. Bathroom activity monitoring based on sound. In Pervasive 2005. 47-61.

[10] Arnaud Doucet. 2001. Sequential Monte Carlo methods. Wiley Online Library.

[11] Tâm Huynh, Mario Fritz, and Bernt Schiele. 2008. Discovery of activity patterns using topic models. In UbiComp 2008. 10-19.

[12] Matthew J. Johnson and Alan S. Willsky. 2013. Bayesian Nonparametric Hidden Semi-Markov Models. Fournal of Machine Learning Research 14, 1 (2013), 673-701.

[13] Oliver Korn, Albrecht Schmidt, and Thomas Hörz. 2013. The potentials of in-situ-projection for augmented workplaces in production: a study with impaired persons. In CHI'13 Extended Abstracts. 979-984.

[14] Joseph Korpela, Ryosuke Miyaji, Takuya Maekawa, Kazunori Nozaki, and Hiroo Tamagawa. 2015. Evaluating tooth brushing performance with smartphone sound data. In UbiComp 2015. 109-120.

[15] Joseph Korpela, Kazuyuki Takase, Takahiro Hirashima, Takuya Maekawa, Julien Eberle, Dipanjan Chakraborty, and Karl Aberer. 2015. An energy-aware method for the joint recognition of activities and gestures using wearable sensors. In International Symposium on Wearable Computers (ISWC 2015). 101-108.

[16] Heli Koskimäki, Ville Huikari, Pekka Siirtola, Perttu Laurinen, and Juha Röning. 2009. Activity recognition using a wrist-worn inertial measurement unit: A case study for industrial assembly lines. In 17th Mediterranean Conference on Control and Automation (MED 2009). 401-405.

[17] Taku Kudo, Kaoru Yamamoto, and Yuji Matsumoto. 2004. Applying conditional random fields to Japanese morphological analysis. In the 2004 Conference on Empirical Methods in Natural Language Processing (EMNLP '04). 230-237.

[18] Jonathan Lester, Tanzeem Choudhury, and Gaetano Borriello. 2006. A practical approach to recognizing physical activities. In Pervasive 2006. 1-16.

[19] Jessica Lin, Eamonn Keogh, Stefano Lonardi, and Pranav Patel. 2002. Finding motifs in time series. In The 2nd Workshop on Temporal Data Mining. 53-68.

[20] Bruno Lotter and Hans-Peter Wiendahl. 2013. Montage in der industriellen Produktion: Ein Handbuch für die Praxis. Springer-Verlag.

[21] Dominik Lucke, Carmen Constantinescu, and Engelbert Westkämper. 2008. Smart factory-a step towards the next generation of manufacturing. In Manufacturing systems and technologies for the new frontier. Springer, 115-118.

[22] Paul Lukowicz, Holger Junker, Mathias Stäger, Thomas von Bueren, and Gerhard Tröster. 2002. WearNET: A distributed multi-sensor system for context aware wearables. In Ubicomp 2002. 361-370.

[23] Paul Lukowicz, Jamie A Ward, Holger Junker, Mathias Stäger, Gerhard Tröster, Amin Atrash, and Thad Starner. 2004. Recognizing workshop activity using body worn microphones and accelerometers. In Pervasive 2004. 18-32.

[24] Takuya Maekawa, Yasue Kishino, Yasushi Sakurai, and Takayuki Suyama. 2013. Activity recognition with hand-worn magnetic sensors. Personal and ubiquitous computing 17, 6 (2013), 1085-1094.

[25] Takuya Maekawa, Yasue Kishino, Yutaka Yanagisawa, and Yasushi Sakurai. 2012. Recognizing handheld electrical device usage with hand-worn coil of wire. In Pervasive 2012. 234-252.

[26] Takuya Maekawa, Yasue Kishino, Yutaka Yanagisawa, and Yasushi Sakurai. 2012. WristSense: wrist-worn sensor device with camera for daily activity recognition. In 2012 IEEE International Conference on Pervasive Computing and Communications Workshops (PERCOM Workshops). 510-512.

[27] Takuya Maekawa, Daisuke Nakai, Kazuya Ohara, and Yasuo Namioka. 2016. Toward practical factory activity recognition: unsupervised understanding of repetitive assembly work in a factory. In UbiComp 2016. 1088-1099.

Proc. ACM Interact. Mob. Wearable Ubiquitous Technol., Vol. 3, No. 2, Article 60. Publication date: June 2019. 
[28] Takuya Maekawa and Shinji Watanabe. 2011. Unsupervised activity recognition with user's physical characteristics data. In International Symposium on Wearable Computers (ISWC 2011). 89-96.

[29] Takuya Maekawa, Yutaka Yanagisawa, Yasue Kishino, Katsuhiko Ishiguro, Koji Kamei, Yasushi Sakurai, and Takeshi Okadome. 2010. Object-based activity recognition with heterogeneous sensors on wrist. In Pervasive 2010. 246-264.

[30] Tomas Mikolov, Ilya Sutskever, Kai Chen, Greg S Corrado, and Jeff Dean. 2013. Distributed Representations of Words and Phrases and their Compositionality. In Advances in Neural Information Processing Systems 26. 3111-3119.

[31] Francisco Javier Ordóñez Morales and Daniel Roggen. 2016. Deep convolutional feature transfer across mobile activity recognition domains, sensor modalities and locations. In The 2016 ACM International Symposium on Wearable Computers (ISWC). ACM, 92-99.

[32] Meinard Müller. 2007. Dynamic time warping. Information retrieval for music and motion (2007), 69-84.

[33] Hamed Pirsiavash and Deva Ramanan. 2012. Detecting activities of daily living in first-person camera views. In CVPR 2012. 2847-2854.

[34] Agnieszka Radziwon, Arne Bilberg, Marcel Bogers, and Erik Skov Madsen. 2014. The Smart Factory: Exploring adaptive and flexible manufacturing solutions. Procedia Engineering 69 (2014), 1184-1190.

[35] Juhi Ranjan and Kamin Whitehouse. 2015. Object hallmarks: Identifying object users using wearable wrist sensors. In UbiComp 2015. 51-61.

[36] N. Ravi, N. Dandekar, P. Mysore, and M.L. Littman. 2005. Activity recognition from accelerometer data. In IAAI 2005, Vol. 20. 1541-1546.

[37] Thomas Stiefmeier, Georg Ogris, Holger Junker, Paul Lukowicz, and Gerhard Tröster. 2006. Combining motion sensors and ultrasonic hands tracking for continuous activity recognition in a maintenance scenario. In 10th IEEE International Symposium on Wearable Computers (ISWC 2006). 97-104.

[38] Thomas Stiefmeier, Daniel Roggen, and Gerhard Tröster. 2007. Fusion of string-matched templates for continuous activity recognition. In 11th IEEE International Symposium on Wearable Computers (ISWC 2007). 41-44.

[39] Edison Thomaz, Irfan Essa, and Gregory D Abowd. 2015. A practical approach for recognizing eating moments with wrist-mounted inertial sensing. In UbiComp 2015. 1029-1040.

[40] Jamie A Ward, Paul Lukowicz, and Gerhard Tröster. 2005. Gesture spotting using wrist worn microphone and 3-axis accelerometer. In The 2005 foint Conference on Smart Objects and Ambient Intelligence: Innovative context-aware services: usages and technologies. 99-104.

Received February 2019; accepted June 2019 